\title{
INTEGRALS OF DYNAMICAL SYSTEMS LINEAR IN THE VELOCITIES
}

\author{
by C. D. COLLINSON
}

(Received 22nd April 1970)

\section{Introduction}

Kilmister (1) has discussed the existence of linear integrals of a dynamical system specified by generalized coordinates $q^{\alpha}(\alpha=1,2, \ldots, n)$ and a Lagrangian

$$
L=\frac{1}{2} a_{\alpha \beta} \dot{q}^{\alpha} \dot{q}^{\beta}+a_{\alpha} \dot{q}^{\alpha}+a,
$$

repeated indices being summed from 1 to $n$. He derived covariant conditions for the existence of such an integral, conditions which do not imply the existence of an ignorable coordinate. Boyer (2) discussed the conditions and found the most general Lagrangian satisfying the conditions for the case of two degrees of freedom $(n=2)$.

In this note it is shown that Kilmister's conditions are too restrictive and a set of necessary and sufficient conditions are obtained. The most general Lagrangian satisfying these conditions is found, without any restriction on the number of degrees of freedom of the system. Finally a comment is made on the "conditional integrals" discussed by Birkhoff (3).

\section{Derivation of the Conditions}

The equations of motion can be written in the covariant form

$$
D \dot{q}^{\alpha} / D t+a^{\alpha \rho}\left(a_{\rho ; \beta}-a_{\beta ; \rho}\right) \dot{q}^{\beta}-a^{\alpha \rho} a_{; \rho}=0,
$$

where the semi-colon denotes covariant differentiation, and $D / D t$ denotes the absolute derivative with respect to the "metric" $a_{\alpha \beta}$ and $a^{\alpha \rho} a_{\rho \beta}=\delta_{\beta}^{\alpha}$. The tensors $a_{\alpha \beta}$ and $a^{\alpha}$ will be used to raise and lower indices in the usual manner. Assume the existence of just one linear integral

$$
b_{\alpha} \dot{q}^{\alpha}=1 \text {. }
$$

The condition for $b_{\alpha} \dot{q}^{\alpha}$ to be constant can be written in the covariant form

$$
b_{\alpha} D \dot{q}^{\alpha} / D t+\frac{1}{2}\left(b_{\alpha ; \beta}+b_{\beta ; \alpha}\right) \dot{q}^{\alpha} \dot{q}^{\beta}=0 .
$$

Using (1) to eliminate the generalized acceleration yields the condition

$$
b^{\rho}\left(a_{\rho ; \beta}-a_{\beta ; \rho}\right) \dot{q}^{\beta}-b^{\rho} a_{; \rho}=\frac{1}{2}\left(b_{\alpha ; \beta}+b_{\beta ; \alpha}\right) \dot{q}^{\alpha} \dot{q}^{\beta} \text {. }
$$

Now assume that (3) is not an identity but is satisfied by virtue of (2). In fact (2) can be used to express (3) as a homogeneous quadratic form in the generalized E.M.S. - $Q$ 
velocities. Comparing coefficients in this quadratic form leads to the condition

$$
b^{\rho}\left(a_{\rho ; \beta}-a_{\beta ; \rho}\right) b_{\alpha}+b^{\rho}\left(a_{\rho ; \alpha}-a_{\alpha ; \rho}\right) b_{\beta}-2 b^{\rho} a_{; \rho} b_{\alpha} b_{\beta}=b_{\alpha ; \beta}+b_{\beta ; \alpha}
$$

for the existence of the linear integral (2). Kilmister's conditions are contained in (4). It is shown later that (4) is a necessary and sufficient condition for the existence of the linear integral (2).

Suppose now that (4) admits a solution $b_{\alpha}$. Consider $u=b_{\alpha} \dot{q}^{\alpha}$. Then a simple calculation, using (4), shows that

$$
\dot{u}=(u-1)\left[b^{\rho}\left(a_{\rho ; \beta}-a_{\beta ; \rho}\right) \dot{q}^{\beta}-(1+u) b^{\rho} a_{; \rho}\right] .
$$

Hence $u=1$ is a singular solution unless

$$
b^{\rho}\left(a_{\rho ; b}-a_{\beta ; \rho}\right)=b^{\rho} a_{; \rho}=0
$$

Substituting (5) into (4) yields

$$
b_{\alpha ; \beta}+b_{\beta ; \alpha}=0
$$

and this is the condition for the existence of an ignorable coordinate.

As Kilmister states, the general situation, when more than one linear integral exists, is far more complicated.

\section{Derivation of the General Lagrangian Admitting a Linear Integral}

It is instructive to split (4) into components along $b_{\alpha}$ and perpendicular to $b_{\alpha}$. The latter components are found by contracting indices with the perpendicular projection operator $h_{v}^{\alpha}=\delta_{v}^{\alpha}-b^{-2} b^{\alpha} b_{v}$ which satisfies $h_{v}^{\alpha} b_{\alpha}=0$. The condition (4) can then be replaced by the following three conditions

$$
\begin{gathered}
b_{(\alpha ; \beta)} h_{v}^{\alpha} h_{\mu}^{\beta}=0, \\
b_{(\alpha ; \beta)} h_{v}^{\alpha} b^{\beta}=b^{\rho} a_{[\rho ; \alpha]} b^{2} h_{v}^{\alpha}
\end{gathered}
$$

and

$$
b_{(\alpha ; \beta)} b^{\alpha} b^{\beta}=-b^{\rho} a_{; \rho} b^{4},
$$

where ( ) and [ ] denote the symmetric and antisymmetric parts respectively.

Suppose now that these equations admit a solution. Then the coordinates can be chosen to make $b^{\alpha}=\delta_{1}^{\alpha}$ so that $b_{\alpha}=a_{\alpha 1}$ and $b_{(\alpha ; \beta)}=\frac{1}{2} a_{\alpha \beta, 1}$. Equation (6) becomes

$$
a_{v \mu, 1}-a_{v 1,1} a_{\mu 1} a_{11}^{-1}-a_{1 \mu, 1} a_{v 1} a_{11}^{-1}+a_{11,1} a_{v 1} a_{\mu 1} a_{11}^{-2}=0,
$$

or

Hence

$$
\left[a_{v \mu}-a_{v 1} a_{\mu 1} a_{11}^{-1}\right]_{, 1}=0
$$

$$
a_{v \mu}=a_{v 1} a_{\mu 1} a_{11}^{-1}+A_{v \mu}
$$


where $A_{v \mu}=A_{\mu v}$ are functions independent of $q^{1}$ with $A_{v 1}=0$. Equation (7) becomes

$$
a_{v 1,1}-a_{11,1} a_{v 1} a_{11}^{-1}=\left(a_{1, v}-a_{v, 1}\right) a_{11}^{-1}
$$

or

Hence

$$
\left(a_{v 1} a_{11}^{-1}+a_{v}\right)_{, 1}=\left(a_{11} a_{11}^{-1}+a_{1}\right)_{, v}
$$

$$
a_{v 1} a_{11}^{-1}+a_{v}=\phi, v+A_{v}
$$

where $A_{v}$ are functions independent of $q^{1}$ with $A_{1} \equiv 0$ and $\phi$ is an arbitrary function. In fact terms involving $\phi$ do not appear in the equations of motion and so, without loss of generality, it can be assumed that

$$
a_{v}=A_{v}-a_{v 1} a_{11}^{-1}
$$

Finally equation (8) yields

$$
a=\frac{1}{2} a_{11}^{-1}+A
$$

where $A$ is a function independent of $q^{1}$. Combining (9), (10) and (11) gives an expression for the most general Lagrangian admitting a linear integral. The Lagrangian can best be written by introducing indices $i, j$, .. which range and sum from 2 to $n$. Then

$$
L=\frac{1}{2} a_{11}^{-1}\left(a_{v 1} \dot{q}^{v}-1\right)^{2}+\frac{1}{2} A_{i j} \dot{q}^{i} \dot{q}^{j}+A_{i} \dot{q}^{i}+A,
$$

where $A_{i j}, A_{j}$ and $A$ are all independent of $q^{1}$. The subcase discussed by Kilmister and Boyer is equivalent to $a_{\nu 1} a_{11}^{-1}$ being independent of $q^{1}$.

\section{Proof that the Condition is Necessary and Sufficient}

Return now to equation (3). Suppose a linear integral exists and choose $b^{\alpha}=\delta_{1}^{\alpha}$. Then the integral can be written as

Hence, since $a_{11} \neq 0$,

$$
a_{\alpha 1} \dot{q}^{\alpha}=1 \text {. }
$$

$$
\dot{q}^{1}=a_{11}^{-1}-a_{i 1} a_{11}^{-1} \dot{q}^{4} .
$$

Using this to eliminate $\dot{q}^{1}$ from (3) yields:

$$
\begin{aligned}
\left(a_{1 ; i}-a_{i ; 1}\right) \dot{q}^{i}-a_{, 1}=\frac{1}{2} a_{i j, 1} \dot{q}^{i} \dot{q}^{j}+a_{1 j, 1} \dot{q}^{j}\left(a_{11}^{-1}-a_{i 1} a_{11}^{-1} \dot{q}^{i}\right) & \\
& +\frac{1}{2} a_{11,1}\left(a_{11}^{-1}-a_{i 1} a_{11}^{-1} \dot{q}^{i}\right)\left(a_{11}^{-1}-a_{j 1} a_{11}^{-1} \dot{q}^{j}\right) .
\end{aligned}
$$

Since this is to be true for all $\dot{q}^{i}$ three equations are obtained. These are necessary and sufficient conditions for the existence of a linear integral and are the same equations as were obtained in the last section. It follows that the covariant condition (4) is both a necessary and sufficient condition for the existence of a linear integral. 


\section{Conditional Linear Integrals}

Birkhoff discusses linear integrals for which (3) is not an identity nor is satisfied by virtue of (2) but is satisfied by virtue of the energy equation. Multiplying the equation of motion (1) by $\dot{q}_{a}$ yields

so that

$$
\dot{q}^{\beta} \dot{q}_{\alpha} \dot{q}_{; \beta}^{\alpha}-\dot{q}^{\rho} a_{; \rho}=0
$$

$$
\frac{d}{d t}\left[\frac{1}{2} a_{\alpha \beta} \dot{q}^{\alpha} \dot{q}^{\beta}-a\right]=0 .
$$

Hence

$$
\frac{1}{2} a_{\alpha \beta} \dot{q}^{\alpha} \dot{q}^{\beta}-a=E
$$

where the constant $E$ is, of course, the total energy. Now (12) can be used to write the condition (3) for a linear integral in the form

$$
b^{\rho}\left(a_{\rho ; \beta}-a_{\beta ; \rho}\right) \dot{q}^{\beta}-\frac{\frac{1}{2} b^{\rho} a_{; \rho}}{E+a} a_{\alpha \beta} \dot{q}^{\alpha} \dot{q}^{\beta}=\frac{1}{2}\left(b_{\alpha ; \beta}+b_{\beta ; \alpha}\right) \dot{q}^{\alpha} \dot{q}^{\beta}
$$

which is satisfied provided that

and

$$
b^{\rho}\left(a_{\rho ; \beta}-a_{\beta ; \rho}\right)=0
$$

$$
\frac{1}{2}\left(b_{\alpha ; \beta}+b_{\beta ; \alpha}\right)=-\frac{b^{\rho} a_{; \rho}}{E+a} a_{\alpha \beta} .
$$

The last equation is familiar in Riemannian geometry (4) and expresses the fact that the vector field $b_{\alpha}$ describes a conformal motion of the metric $a_{\alpha \beta}$. The most general Lagrangian admitting a conditional linear integral can be written in the form (i.e. choosing $b^{\alpha}=\delta_{1}^{\alpha}$ )

$$
L=\frac{1}{2} A_{\alpha \beta} \dot{q}^{\alpha} \dot{q}^{\beta}(E+a)^{-1}+A_{\alpha} \dot{q}^{\alpha}+a
$$

where $A_{\alpha \beta}$ and $A_{\alpha}$ are independent of the coordinate $q^{1}$.

\section{REFERENCES}

(1) C. W. KILMISTER, The existence of integrals of dynamical systems linear in the velocities, Edinburgh Math. Notes 44 (1961), 13-16, in Proc. Edinburgh Math. Soc. (2) 12 (1960-61).

(2) R. H. BOYER, On Kilmister's conditions for the existence of linear integrals of dynamical systems, Proc. Edinburgh Math. Soc. 14 (1964), 243-244.

(3) G. D. Birkнoff, Dynamical Systems (New York, 1927), p. 44.

(4) L. P. Eisenhart, Riemannian Geometry (Princeton University Press, 1925), p. 230 .

Department of Applied Mathematics

THE UNIVERSITY, HULL 